\title{
Express Saccades Elicited During Visual Scan in the Monkey
}

\author{
M. A. SOMMER* \\ Received 23 February 1993; in revised form 20 August 1993; in final form 24 November 1993
}

\begin{abstract}
Monkeys trained to saccade to visual targets can develop separate "express" and "regular" modes in their distribution of saccadic latencies. The purpose of this study was to determine whether this occurs under more natural viewing conditions, when targets are suddenly presented in a structured visual field during visual scan. It was found that scanning saccades stopped appearing $60 \mathrm{msec}$ after a target's onset, and subsequent saccades, which were directed toward the suddenly appearing target, had a bimodal distribution of latencies. Express saccades were more likely to occur as the target was presented later in a fixation. Regular mode saccades were more likely to occur with longer target durations. Scanning saccades made to stimuli of the structured visual field always had unimodal inter-saccadic interval distributions. All these effects were apparent after only $2-3$ days of training. These findings, taken together with recent physiological results, suggest that the visuomotor cells of the superior colliculus mediate latency bimodality.
\end{abstract}

Rhesus monkey Saccidic eye movements Express saccades Visual scan Superior colliculus

\section{INTRODUCTION}

When a primate's task is to make a saccadic eye movement toward a novel visual stimulus, saccadic latencies can form a bimodal distribution (Fischer \& Boch, 1983). In the monkey, for example, saccades can be initiated either about $80 \mathrm{msec}$ or after $120 \mathrm{msec}$ following target onset, forming "express" and "regular" latency modes, respectively, but few saccadic latencies occur around $100 \mathrm{msec}$.

In previous studies, the procedure for evoking express saccades has required a subject to fixate a point in the center of his visual field before presenting a saccade target in the featureless periphery. A temporal "gap" has been introduced by removing the fixation point a short time (typically 100 or $200 \mathrm{msec}$ ) prior to target onset in almost every experiment (a short list includes Fischer \& Boch, 1983; Boch, Fischer \& Ramsperger, 1984; Boch \& Fischer, 1986; Smit \& Van Gisbergen, 1989; Weber \& Fischer, 1990; Jüttner \& Wolf, 1992; Weber, Latanov \& Fischer, 1993; Nothdurft \& Parlitz, 1993). This traditional paradigm, however, does not represent everyday conditions encountered by primates. Natural visual scenes are generally structured, and thus any sudden visual change that might elicit a saccade usually occurs amidst other background, non-target stimuli. Only two previous studies (Mayfrank, Mobashery, Kimmig \& Fischer, 1986; Schiller, Sandell \& Maunsell, 1987) have

*Department of Brain and Cognitive Sciences, Massachusetts Institute of Technology, Cambridge, MA 02139, U.S.A. [Email alois@athena.mit.edu]. considered any effects of non-target visual stimuli on express saccades. Also, humans and monkeys normally scan such structured visual fields, moving their eyes at 2-5 saccades/sec with saccades ranging from 2 to $15 \mathrm{deg}$ in amplitude (Bahill, Adler \& Stark, 1975; Schiller, True \& Conway, 1980).

Can express latency saccades occur under conditions that involve visual structure and scanning? This is important to know, in order to understand whether express saccades might be used by primates in everyday situations. Aside from a brief comment in an early paper (Fischer, Boch \& Ramsperger, 1984), there have been no prior reports of attempts to determine whether express latencies occur under more natural conditions than the traditional ones described above. The present study is the first quantified effort in this direction.

In the paradigm of this report, monkeys are presented with a structured visual field. They make successive saccadic eye movements to the stimuli that comprise this field. Target stimuli are presented at random times and positions during natural fixations of the visual scan; the task is to saccade directly to the suddenly appearing target. If express latency saccades $d o$ occur using this paradigm, the finding would suggest that monkeys are indeed capable of foveating suddenly appearing visual stimuli with express saccades during normal visual scanning of their environment. This would be a significant step toward demonstrating that express saccades are a normal element of a monkey's oculomotor repertoire. A negative finding, that express saccades do not occur during this "Visual Scan" paradigm, would also be 
interesting, since determining the causes of this inability could shed more light on the mechanisms underlying express latencies.

In the course of executing the experiments described in this report, I also examined the effects of two aspects of the target's timing on elicitation of express and regular latency saccades: the target onset lag (i.e. the asynchrony between start of fixation and appearance of the target) and the target duration. Target onset lag effects on express saccades have never been studied before; although many investigators have varied the duration of the fixation stimulus (Fischer \& Boch, 1983; Fischer \& Ramsperger, 1984, 1986; Rohrer \& Sparks, 1986; Boch \& Fischer, 1986; Kalesnykas \& Hallet, 1987; Schiller et al., 1987; Smit \& Van Gisbergen, 1989; Fischer, Weber \& Biscaldi, 1993; Nothdurft \& Parlitz, 1993), the actual durations of foveation of the fixation stimulus have not been reported. This study synchronizes all target onsets to the actual acquisition of the fixation stimulus and uses five randomized target onset lags to probe how length of fixation affects saccadic latency. Also, during early testing sessions it was noticed that very short target durations markedly reduced the animals' ability to perform the tasks. This decrement in the frequency of correct trials as target duration decreased was due solely to a loss of longer latency saccades. Since an earlier paper (Boch et al., 1984) had reported no effect of target duration on bimodal saccadic latency distributions, the target duration effects observed in the present study were thoroughly investigated.

\section{METHODS}

\section{Subjects}

Two rhesus monkeys (Macaca mulatta) were the subjects for these experiments. Each animal underwent surgery for the implantation of a head post and scleral search coil (Robinson, 1963; Fuchs \& Robinson, 1966). Surgery was performed in sterile conditions, with animals under sodium pentobarbitol anesthesia. Animals received analgesics and antibiotics for several days following the surgery, and training on oculomotor tasks began 2 weeks after recovery. Monkeys were deprived of water in their home cage but were rewarded with apple juice during testing and allowed to drink until satiated after testing. All animal use followed NIH guidelines.

One subject, monkey $\mathrm{C}$, had never been used in any experiment before this study. Monkey $\mathrm{D}$ was initially naive to this study's paradigm, and had not been trained previously to produce express saccades.

\section{Computers and equipment}

Each monkey sat in a primate chair $65 \mathrm{~cm}$ from a $60 \mathrm{~Hz}$ interlaced color monitor. A PDP-11 computer drove the graphics, ran the real-time stimulus presentation programs, and collected the data. Eye position samples and task event information were stored every $5 \mathrm{msec}$. The controlling program waited for vertical retrace of the monitor before drawing a target, to ensure accurate presentation.

\section{Timing of all trials}

A fixation spot appeared to start an experimental trial and was continuously lit until the trial ended (Fig. 1). If an animal did not foveate the fixation stimulus within $10 \mathrm{sec}$, the trial was aborted and a new trial began after the inter-trial interval of $1500 \mathrm{msec}$. If the subject's eye position did enter a computer-defined "window" near the fixation spot, and if it remained there for a minimum time, collection of eye position samples commenced. The program did not present a target immediately but continued to wait during a "target onset lag" period. The trial was aborted if the eye position left the fixation window during the target onset lag. If the trial was not aborted, a target would then be fiashed for a certain duration. The length of time from target onset to initiation of a correct saccadic eye movement was the saccadic latency (determined off-line). A direct saccade that terminated in a window around the target would be rewarded with a drop of juice. Randomly, one out of three incorrect responses were rewarded to keep the animal from quitting during difficult tasks. Note that all trials were the "overlap" type, i.e. fixation stimuli remained on throughout target presentation.

Target windows measured $2.8 \times 2.8 \mathrm{deg}$, i.e. $4 \mathrm{deg}$ diagonally. The fixation window sizes were chosen to balance two considerations: the window had to be big enough that most foveations of the fixation stimulus occurred within it, but it had to be small enough that most saccades passing through the window would not remain within it long enough to be erroneously judged a fixation. Fixation windows for monkey $\mathrm{D}$ were $2.8 \times 2.8 \mathrm{deg}$, and the minimum fixation time (Fig. 1) for this animal was $30 \mathrm{msec}$. To achieve shorter target onset lags for monkey $\mathrm{C}$ (see Task parameter selection, below), the minimum fixation time was set to $10 \mathrm{msec}$ and the fixation windows were set to $2.0 \times 2.0 \mathrm{deg}$. All window

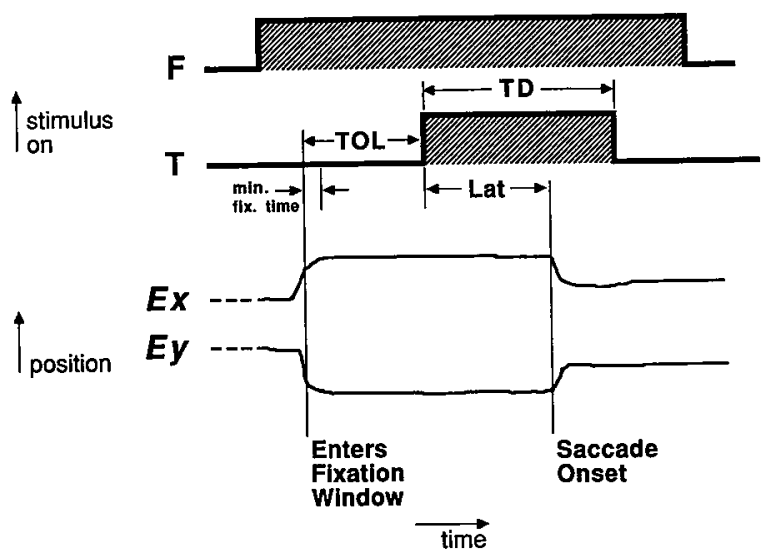

FIGURE 1. Timing diagram for all trials of the paradigm. A fixation spot (F) appears to begin the trial. The eye position (components $E x$, horizontal, and $E y$, vertical) enters a fixation window and remains for a minimum length of time (min. fix. time). After a target onset lag (TOL) period, the target (T) is illuminated for a target duration (TD). The time that elapses after target onset until the monkey initiates a saccade to the target is the latency (Lat). 


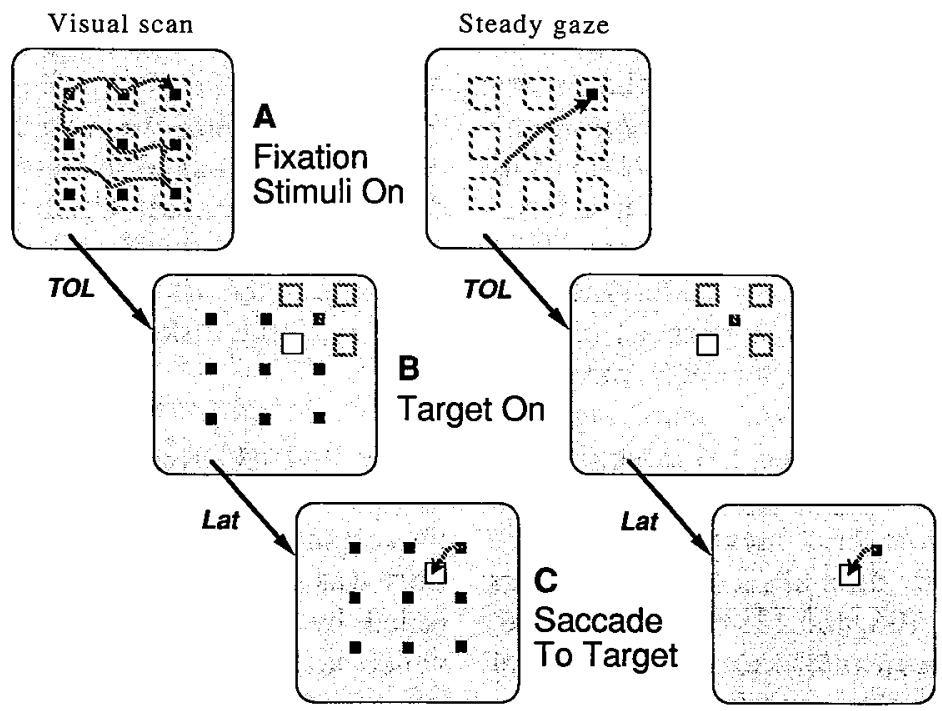

FIGURE 2. Spatial aspects of each task. Heavy broken curve represents eye movements, broken squares around stimuli depict windows (not seen by monkey). (A, left) In the Visual Scan task, a trial begins with the appearance of fixation stimuli at nine positions on the monitor. Monkeys scan the stimulus array with saccades. (B, left) Foveation of one of these spots triggers target onset in one of four randomized positions around the fixation position. (C, left) A direct saccade to the target results in juice reward. (A, right) In the Steady Gaze task, only one fixation stimulus appears to begin a trial. Monkeys foveate this spot immediately. (B, C, right) All subsequent trial events are identical to those of the Visual Scan task. TOL, target onset lag; Lat, saccadic latency.

sizes and minimum fixation times were established during early training of each monkey and were not changed thereafter.

\section{Spatial aspects of trials}

The fixation stimuli were $0.3 \times 0.3 \mathrm{deg}$ black squares, the target stimulus was a $0.6 \times 0.6 \mathrm{deg}$ white $\left(71 \mathrm{~cd} / \mathrm{m}^{2}\right)$ square, and the background was gray $\left(9 \mathrm{~cd} / \mathrm{m}^{2}\right)$. Dim ambient light in the testing room allowed objects such as the monitor, monitor stand, and walls to be seen; hence, the black squares on the monitor provided a local structure within a larger unchanging visual scene. The fixation spot array (Fig. 2, left) over which visual scan occurred was drawn so that the center stimulus was directly ahead of the monkey. This $3 \times 3$ array measured $16.0 \mathrm{deg}$ diagonally. All targets were presented $4.0 \mathrm{deg}$ eccentric to the foveated fixation stimulus, obliquely at either $45,135,225$, or $315 \mathrm{deg}$ meridian from horizontal. A target presented within the array would be centered between four fixation stimuli [e.g. Fig. 2(B, C) left].

\section{Visual Scan task}

At the start of a Visual Scan trial, an array of fixation point stimuli appeared on the monitor [Fig. 2(A), left]. Although the fixation stimuli were all identical in appearance, the computer program randomly pre-selected one of these stimuli to be the "trigger" for target onset. Monkeys responded to presentation of the array by performing a rapid visual scan over the array. Note that an animal was free to foveate any of the fixation stimuli, in any order, and for any length of time; the only time constraint was the previously mentioned $10 \mathrm{sec}$ trial limit. Once the pre-selected trigger fixation stimulus was foveated, a target onset lag ensued and the target was presented [Fig. 2(B, C), left]. A typical eye movement trace generated by a monkey successfully performing a Visual Scan task trial is shown in Fig. 3(A).

\section{Steady Gaze task}

Since it was not known a priori whether express saccades would occur during the Visual Scan task, a negative finding could have meant either that (1) the Visual Scan task itself inhibited express saccades, or that (2) the monkeys just were not capable of making express saccades or were not sufficiently trained. Therefore, control trials were used to determine whether the monkeys could even make express saccades in the more traditional task. These trials were termed "Steady Gaze" because no scanning was involved. The computer program implemented this task by running a Visual Scan trial with only the pre-selected "trigger" fixation stimulus drawn [Fig. 2(A), right]. An animal would saccade directly to this fixation stimulus and trigger target onset [Fig. 2(A, B, C), right]. Figure 3(B) shows a typical eye movement trace from a Steady Gaze trial.

\section{Inter-saccadic intervals measurements}

Eye position samples were usually not recorded during the saccades of visual scan that occurred before the trigger fixation stimulus was foveated. In order to estimate the durations of the pauses between scanning saccades, the program recorded, on-line, every time $a^{\prime}$ subject's eye position entered or left each fixation window. The amount of time spent in each fixation window could then be calculated from these data, resulting in estimates of the inter-saccadic intervals.

\section{Task parameter selection}

Stimulus parameters were randomized in time and space to minimize anticipatory responses. Location of a 
trial's pre-selected trigger fixation stimulus was pseudorandomized, as was target position, such that every fixation/target position combination would occur in one "block". Thus each block of testing consisted of 36 trials ( 9 trigger fixation positions $\times 4$ target positions). Independent of these spatial randomizations, for each trial one of five target onset lags was pseudo-randomly selected. These target onset lag values were set in early training to probe comparable periods in each monkey's mean inter-saccadic interval. Target onset lags for monkey D were $57,76,101,126$, and $151 \mathrm{msec}$ (i.e. 29, $39,52,64$, and $77 \%$ of its mean inter-saccadic interval of $196 \mathrm{msec}$ ). Due to a more brief mean inter-saccadic interval of monkey $\mathrm{C}$, this animal's target onset lags were shorter, at $36,56,81,106$, and $131 \mathrm{msec}$ (i.e. 23, 36, 52,68 , and $84 \%$ of its mean inter-saccadic interval of $156 \mathrm{msec})$. The 36 spatial $\times 5$ temporal parameters pro-

(A)
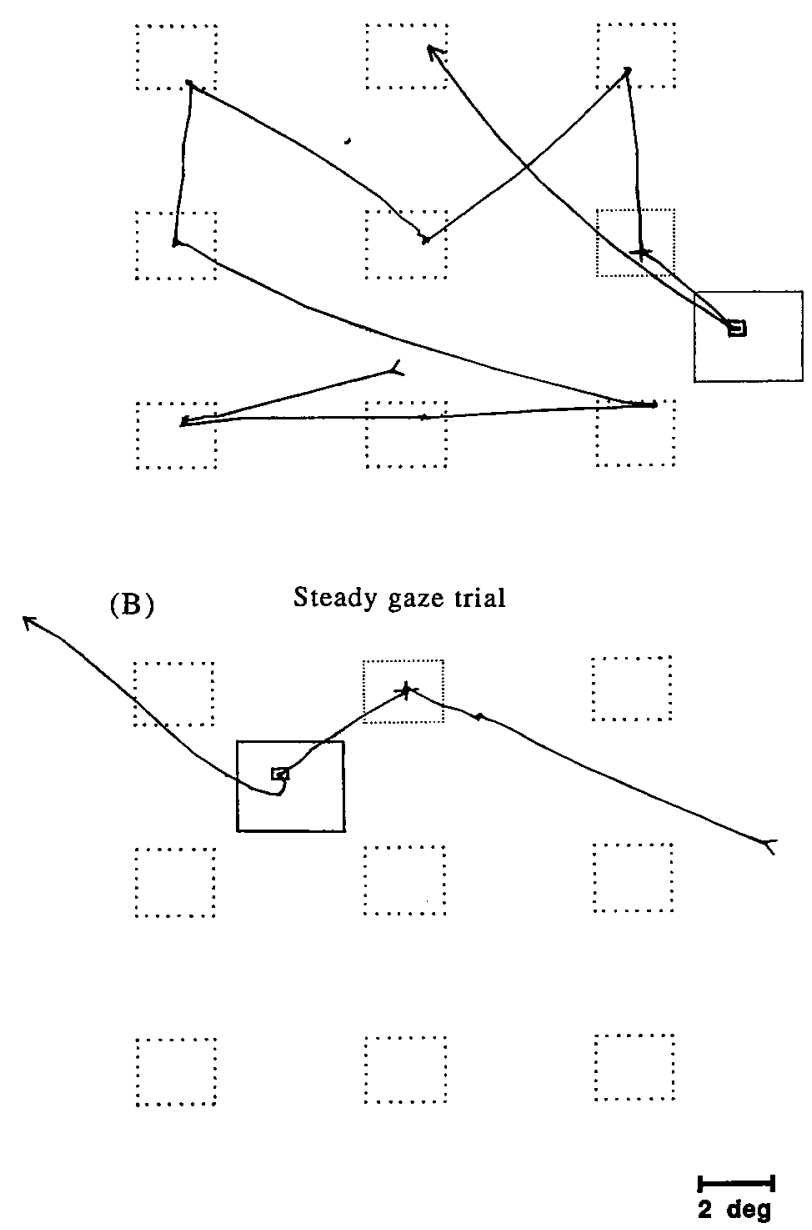

FIGURE 3. Typical eye movement traces of monkey " $C$ ", during the (A) Visual Scan and (B) Steady Gaze tasks. Eye position samples have been connected with straight lines for clarity. Arrowheads show start and end of trace, as well as direction of movement. Windows around each fixation spot location are drawn with dotted lines, and the trigger fixation spot's window is depicted with a finer grain. Largest square represents window around the target; for juice reward, the monkey had to saccade directly into the target window. A small cross and small square mark the initial and final positions, respectively, of the first eye movement that occurred after entering the trigger fixation window. Spatial scale is shown at lower right. vided a total of 180 -fold spatio-temporal randomization, by trial.

Target duration was constant during each set of five blocks, then changed. Unless noted otherwise, six target durations were used: $33,50,67,83,100$, and $150 \mathrm{msec}$. For monkey D, the Visual Scan and Steady Gaze tasks were alternated after each set of five blocks, whereas for monkey $\mathrm{C}$ the tasks were alternated randomly by trial.

\section{Off-line data analysis}

The first $640 \mathrm{msec}$ (128 samples) of the eye position data of each trial was analyzed. Only the first eye movement of a trial was characterized for this report [this was to avoid considering saccades that were preceded by other movements, the refractory periods of which could affect saccadic latency (Carpenter, 1988)]. A change in mean eye position, computed as a running sum over six samples, that exceeded $0.25 \mathrm{deg}$ was considered an eye movement. If such a movement also exceeded $50 \mathrm{deg} / \mathrm{sec}$ at peak velocity it was judged to be a saccade; movements with a smaller peak velocity could have involved mechanisms of the smooth pursuit system (Fuchs, 1967) and were considered non-saccadic eye movements. Saccade start and end times were determined in the velocity domain using a $30 \mathrm{deg} / \mathrm{sec}$ crossing threshold, and saccadic initial and final positions were calculated as an average of six sample positions before or after the movement.

\section{RESULTS}

The basic steady state results will be reported first. Then I will describe changes that occurred during the short-term training that led to steady state. Finally, preliminary observations of long-term training effects will be noted.

\section{Aborted trials}

The $10 \mathrm{sec}$ maximum time limit for acquiring the pre-selected fixation point was hardly ever exceeded in the Visual Scan task (Table 1) and it was never exceeded in the Steady Gaze task. Trials that were aborted because the pre-selected fixation stimulus was foveated, but then the eye position left the fixation window before the full target onset lag occurred, were more common (Table 1: Visual Scan task, $18.4 \%$ of all trial initiations for monkey C, $7.4 \%$ for monkey D, Steady Gaze task, $8.6 \%$ of all trial initiations for monkey C, $1.8 \%$ for monkey D). In the Visual Scan task, these types of aborts occurred nearly always because a scanning saccade was initiated. In the Steady Gaze task, there were three reasons a trial was aborted. The most common reason was that the initial fixation landed near the edge of the electronic window and the slight inherent jitter of the eye position moved the eye trace momentarily outside the window. Secondly, a slow movement sometimes remained within a fixation window long enough to be judged a fixation but then landed just outside the window. Finally, a movement was sometimes made that brought the eye position out of the fixation window, 
TABLE 1. Breakdown of the results of attempting to present a target, for each monkey in each task

\begin{tabular}{|c|c|c|c|c|}
\hline \multirow{2}{*}{$\begin{array}{l}\text { Results of attempts } \\
\text { to present target }\end{array}$} & \multicolumn{2}{|c|}{ Visual scan } & \multicolumn{2}{|c|}{ Steady gaze } \\
\hline & Monkey C & Monkey D & Monkey C & Monkey D \\
\hline $\begin{array}{l}\text { (1) Aborted, target not presented } \\
\text { (exceeded } 10 \mathrm{sec} \text { maximum } \\
\text { acquisition time of fix-spot) }\end{array}$ & $0.3(10 / 3171)$ & $1.7(39 / 2335)$ & $0.0(0 / 2893)$ & $0.0(0 / 2197)$ \\
\hline $\begin{array}{l}\text { (2) Aborted, target not presented } \\
\text { (left fix-spot window during } \\
\text { target onset lag) }\end{array}$ & $18.4(582 / 3171)$ & $7.4(172 / 2335)$ & $8.6(250 / 2893)$ & $1.8(39 / 2197)$ \\
\hline (3) Non-aborted, target presented & $81.3(2579 / 3171)$ & $91.0(2124 / 2335)$ & $91.4(2643 / 2893)$ & $98.2(2158 / 2197)$ \\
\hline
\end{tabular}

See text for details. Table entries are percentages of all trial initiations, derived from the ratios in parentheses; percentages may not sum to 100.0 , due to round-off error.

toward a target location. These possibly anticipatory responses were the rarest type of abortive movement and nearly always terminated far ( $>3 \mathrm{deg}$ ) from any of the four potential target locations surrounding the fixation stimulus.

The great majority of trials of both tasks were not aborted, allowing for target presentation (Table 1). Non-aborted trials are the focus of the rest of this paper. More than 2100 target presentations during each task were tested on each monkey.

\section{A proximity transition with latency}

In Fig. 4, the proximity of the first saccade of nonaborted trials to the center of the suddenly appearing target is plotted against the saccade's latency; superimposing data from many trials results in a scatter plot. The first main result of this report is that a transition in proximity with latency is evident for the Visual Scan task [Fig. 4(A)]. Saccades that were initiated before $60 \mathrm{msec}$ following sudden target onset generally land far ( $>3 \mathrm{deg}$ ) from the target location, but saccades with latencies of $60 \mathrm{msec}$ or longer land very near $(<2 \mathrm{deg})$ to the target location [Fig. 4(A)]. The transition zone is about $20 \mathrm{msec}$ in width, from 50 to $70 \mathrm{msec}$ latency. Many saccades with latencies within this transition zone appear to undergo spatial averaging, since the shift in proximity from 50 to $70 \mathrm{msec}$ is gradual. This transition is also observed in the Steady Gaze task [Fig. 4(B)], but it is especially striking in the Visual Scan task because saccades are continually being elicited throughout the transition zone.

Saccades are separated into two classes for the remainder of this report, by a proximity criterion. If the first movement of a trial is a saccade that has a final proximity nearer to the target than to any of the fixation stimuli or the edge of the visible display, it is termed a "correct" saccade (crosses in Fig. 4) and the trial is a "saccade correct" trial. Otherwise, the saccade is "wrong" (circles in Fig. 4), and the trial is a "saccade wrong" trial.

\section{Types of trial outcomes}

Saccade correct trials. Table 2 shows that the first eye movement of non-aborted trials was a correct saccade at about the same frequency for both monkeys in Steady Gaze trials (59.9 and 55.4\% for monkeys C and D respectively). Monkey D was more proficient at making correct saccades in the Visual Scan trials than monkey C $(58.3 \%$ vs $39.4 \%)$.

The second main result of this study is that latencies of the correct saccades formed a bimodal distribution in both the Visual Scan and Steady Gaze tasks for both monkeys [Fig. 5(B, D)]: an earlier "express" mode was centered between 50 and $100 \mathrm{msec}$ latency, and a later "regular" latency mode appeared after $100 \mathrm{msec}$. Correct saccade trajectories were occasionally curved in the Visual Scan task [Fig. 5(A)], but the accuracy of correct saccade final positions in the Visual Scan task was comparable to the accuracy in the Steady Gaze task [Fig. 5(C)]. For both tasks, nearly all saccades of both monkeys landed within 2 of the target's center (with most within $1 \mathrm{deg}$ ).

Saccade wrong trials. Wrong saccades were more than twice as common in the Visual Scan task than in the Steady Gaze task (Table 2). Figure 6 shows the trajectories and latency distributions of wrong saccades. Trajectories of wrong saccades in the Visual Scan task [Fig. 6(A)] demonstrate that most of these were scanning saccades. The latency histogram of wrong saccades from Visual Scan trials [Fig. 6(B)] reflects the effect seen in Fig. 4(A): the majority of wrong saccades in the Visual Scan task appeared from just before target onset until about $60 \mathrm{msec}$ after target onset. A second, widely scattered mode of wrong saccades appeared after $200 \mathrm{msec}$ latency; these typically terminated off the visible display.

In the Steady Gaze task, wrong saccades were generally corrective movements that improved on inaccurate initial fixations [Fig. 6(C)]. These occurred before $60 \mathrm{msec}$ or so following target onset [Fig. 6(D)]. A small peak of wrong saccades at around $80 \mathrm{msec}$ represent apparently hypometric express saccades which are also evident in Fig. 4(B). As in the Visual Scan task, saccades in the Steady Gaze task that occurred with more than $200 \mathrm{msec}$ or latency generally went off the visible display.

\section{Target onset lag effect}

The third main result of this study is that the express mode of latency distributions of correct saccades is more prominent if the target is presented later in the fixation period (Fig. 7; normalized ordinate is number of saccades divided by number of target presentations, giving 


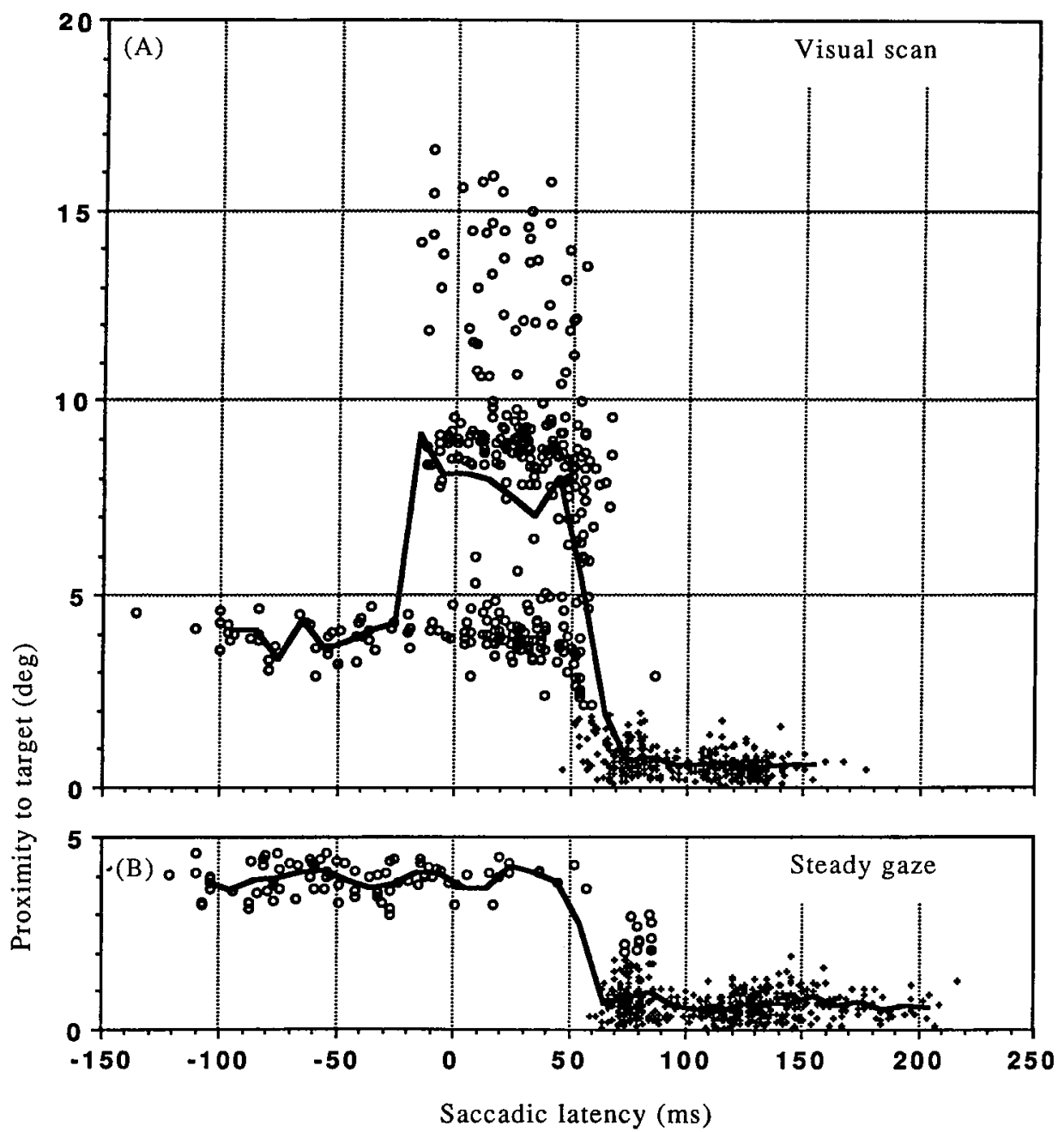

FIGURE 4. Proximity transition with latency. For saccadic first movements of non-aborted trials, each saccade's final proximity to the target is plotted against its latency (both monkeys pooled, all target onset lags pooled, target duration $150 \mathrm{msec}$ ). Zero on the abscissa marks the time of target onset. The heavy line is a running mean, constructed by averaging the proximities over each $10 \mathrm{msec}$ latency interval and assigning that mean to the middle of the interval, then connecting these means with straight lines. Crosses represent "correct" saccades, those with closer final proximity to the target than to any fixation stimulus or edge of the visible display. Circles are all other, "wrong", saccades. (A) A sharp transition in proximity occurs about $60 \mathrm{msec}$ following target onset in Visual Scan trials: the mean proximity to the target drops from around 8 deg during scanning ( $0-45 \mathrm{msec}$ latency), to near $2 \mathrm{deg}$ at $65 \mathrm{msec}$ latency, stabilizing at $<1$ deg by $75 \mathrm{msec}$. (B) A similar transition is seen in the Steady Gaze trials, with a drop in proximity from around 4 to $\leqslant 1$ deg during the interval from 45 to $65 \mathrm{msec}$ latency. The few saccades that landed outside of the visible display are excluded from this figure.

overall frequency of saccade occurrence). The target onset lag effect is apparent for both the Visual Scan [Fig. 7(left)] and the Steady Gaze [Fig. 7(right)] tasks. During the Visual Scan task, the probability of evoking any correct saccade at all with a target presentation decreases as target onset lag increases, because there is a greater chance that a scanning, "wrong", saccade will occur after longer target onset lags.

The target onset lag effect is quantified in Fig. 8, to show that the ratio of express saccades to all correct saccades rises steeply for both the Visual Scan and Steady Gaze tasks, for both monkeys, from a mean of

TABLE 2. Types of the first eye movements in all non-aborted trials and the frequency of occurrence of each type, for each monkey in each task

\begin{tabular}{lcclcc}
\hline \multirow{2}{*}{$\begin{array}{l}\text { First eye movement of } \\
\text { non-aborted trials }\end{array}$} & \multicolumn{2}{c}{ Visual scan } & & \multicolumn{2}{c}{ Steady gaze } \\
\cline { 2 - 3 } \cline { 5 - 6 } \cline { 5 - 6 } (1) Saccade correct & Monkey C & Monkey D & & Monkey C & Monkey D \\
(2) Saccade wrong & $39.4(1016 / 2579)$ & $58.3(1239 / 2124)$ & & $59.9(1583 / 2643)$ & $55.4(1196 / 2158)$ \\
(4) Non-saccadic eye movement & $56.6(1461 / 2579)$ & $40.0(850 / 2124)$ & & $23.3(617 / 2643)$ & $11.9(257 / 2158)$ \\
(3) No eye movement detected & $1.0(27 / 2579)$ & $1.1(23 / 2124)$ & & $8.5(224 / 2643)$ & $27.1(585 / 2158)$ \\
\hline
\end{tabular}

Table entries are percentages of non-aborted trials, derived from the ratios in parentheses; percentages may not sum to 100.0 , due to round-off error. 
Saccade correct: visual scan

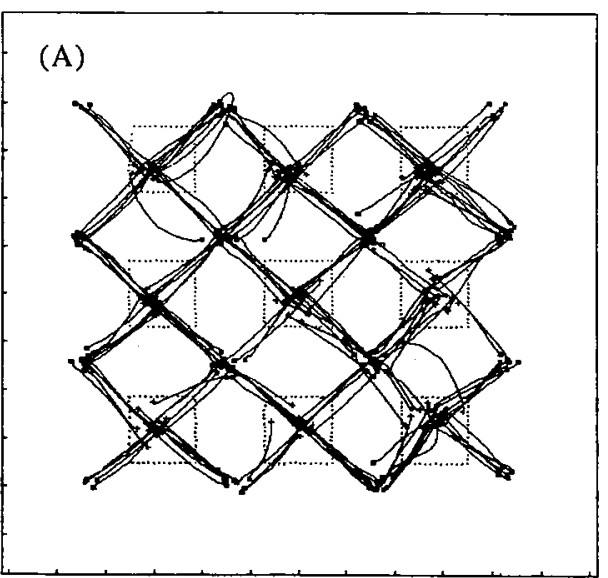

Saccade correct: steady gaze

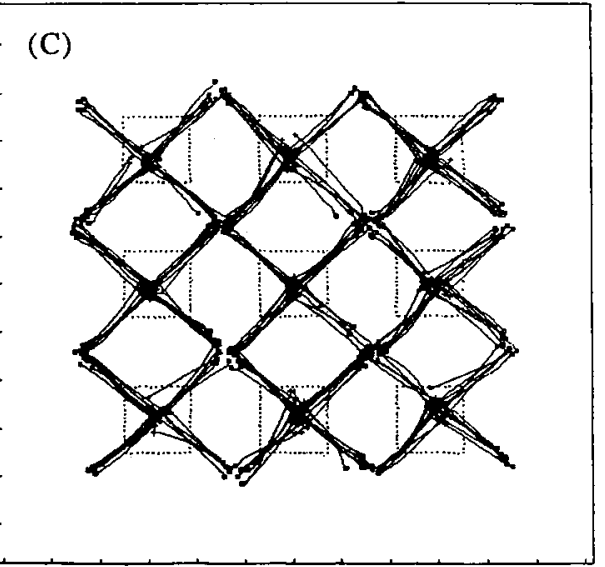

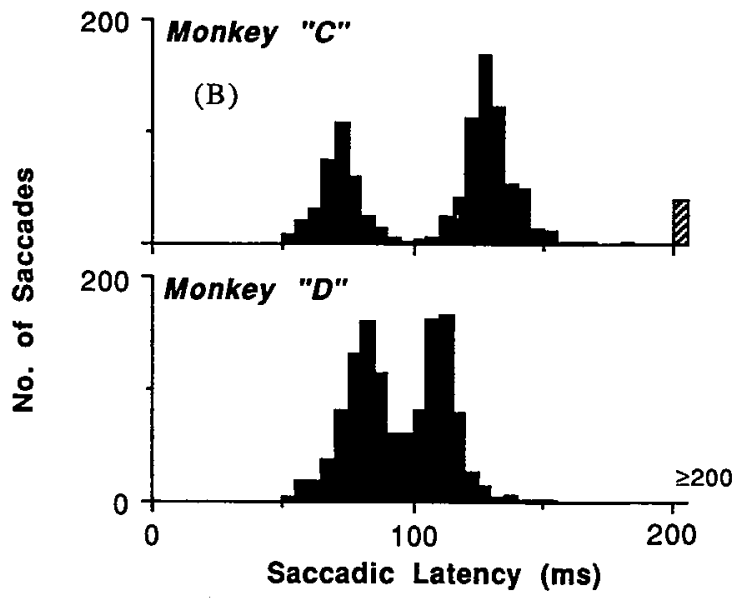

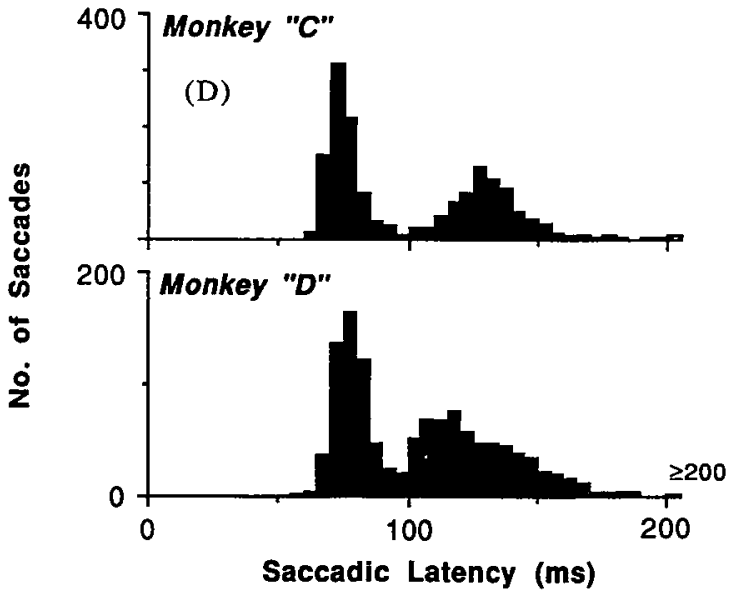

FIGURE 5. Trajectories and latencies of correct saccades. (A) Example of trajectories of correct saccades made in the Visual Scan task. A few saccades exhibit a curved trajectory, but most go directly to the target location. The saccade trace for each trial is drawn as described in Fig. 3: a small cross marks the initial position of the movement, a small square marks the final position, and the eye position samples in between are connected by straight lines. The nine large dotted squares show windows around the fixation spots. The frame around the eye movements represents the edge of the visible display, and ticks on the frame are spaced by 2 deg. (B) Latency distributions of all the correct saccades for monkey "C" (top) and monkey "D" (bottom) in the Visual Scan task. For both monkeys, the latency distributions were bimodal. (C) Trajectories of correct saccades in the Steady Gaze task. (D) Bimodal latency distributions of correct saccades for each monkey in the Steady Gaze task. Saccade traces in (A) and (C) are of monkey "D"; for clarity, only the $150 \mathrm{msec}$ target duration trials are shown. Histograms in (B) and (D) pool together data from all target onset lags and target durations for each monkey.

$27 \%$ at the shortest target onset lag to a mean of $68 \%$ at the longest target onset lag. This increase is highly significant (linear regression of percent express saccades against the independent variable, normalized target onset lag, gives $R^{2}>0.93$ for all four data sets).

\section{Target duration effects}

The fourth and final main result of this study is that the ability to execute a correct saccade is directly proportional to the duration of the flashed target in the Steady Gaze task, and this improvement in performance is due to modulation of the regular mode alone [Figs 9 and 10 (right column of each)]. In contrast, the probability of evoking express saccades was relatively unaffected by the target duration. This target duration effect, although consistently strong in the Steady Gaze task, was less clear in the Visual Scan task [apparent.for monkey C, Fig. 10(A), but not monkey D, Fig. 9 (left) and Fig. 10(B)].

\section{Short-term training effects}

Monkey $\mathrm{C}$, initially naïve, was first trained to fixate a visual stimulus for juice reward and then to saccade to a peripheral visual target. The first day that the monkey $\mathrm{C}$ performed this latter, saccade-to-target, task, the animal was introduced to the Visual Scan and Steady Gaze tasks and testing began. This was Day 1 for monkey C. Day 1 for monkey D, initially familiar with saccade-to-target tasks, was the first day of testing on the Visual Scan and Steady Gaze tasks.

An express mode appeared for both animals by Day 2 of training, for both the Steady Gaze and Visual Scan tasks [monkey $\mathrm{C}$ results are illustrated in Fig. 11(A)]. The target onset lag effect was also established on Day 
2 for both animals [cf. Fig. 11(B), with Fig. 8 monkey $\mathrm{C}$ results]. Varying the target duration for monkey $\mathrm{C}$ first began on Day 2, and the target duration effect was clear on that day [cf. Fig. 11(C), with Fig. 10 monkey C results]. Examination of early training influence on the target duration effect in monkey $\mathrm{D}$ was not possible, because this animal performed fewer trials per day during training.

The mean and variance of each monkey's intersaccadic intervals decreased during training [Fig. 12(A), monkey $\mathrm{C}$; results of monkey $\mathrm{D}$ were similar]. This training effect paralleled the effects shown in Fig. 11; thus, all effects reported in this paper appeared within the first days of training and changed only gradually after that. The distributions of inter-saccadic intervals were always unimodal [a steady-state example is shown in Fig. 12(B)], even if the distribution encroached into the $<100 \mathrm{msec}$ or so "express" region of latencies.

\section{Long-term training effects}

Preliminary results suggest that the effects of target duration, but not of target onset lag, can be modified by extensive training. Following 4 months of daily training, monkey $\mathrm{D}$ now performs as well at short target durations as at long ones. Also, no effects of target duration were found in two other monkeys used in a pilot study of this experiment, both of which had been extensively trained to produce express saccades prior to exposure to this paradigm. Monkey $\mathrm{C}$, however, still exhibits a strong target duration effect, even after 4 months of daily experiments. Thus, it seems that for some monkeys the response to short duration targets can improve over extensive training. In contrast, the target onset lag effects were observed in all monkeys, regardless of prior training, and they did not diminish with further extensive training.

\section{Saccade wrong: visual scan}
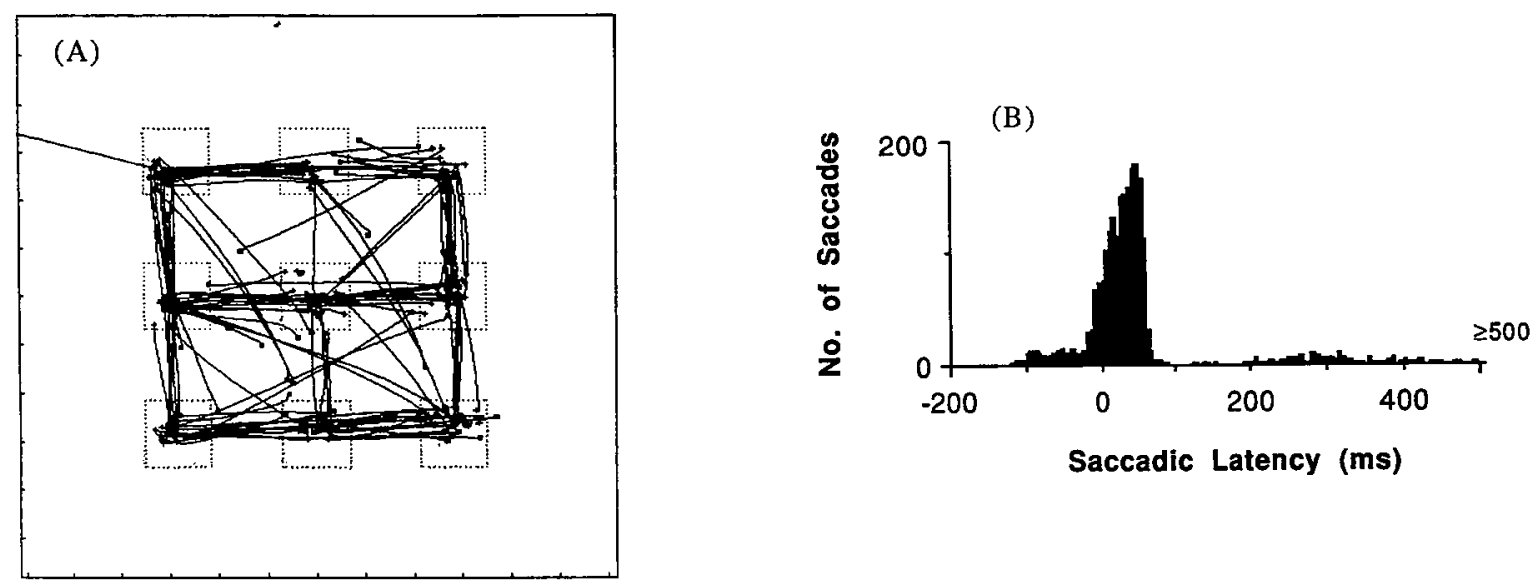

Saccade wrong: steady gaze
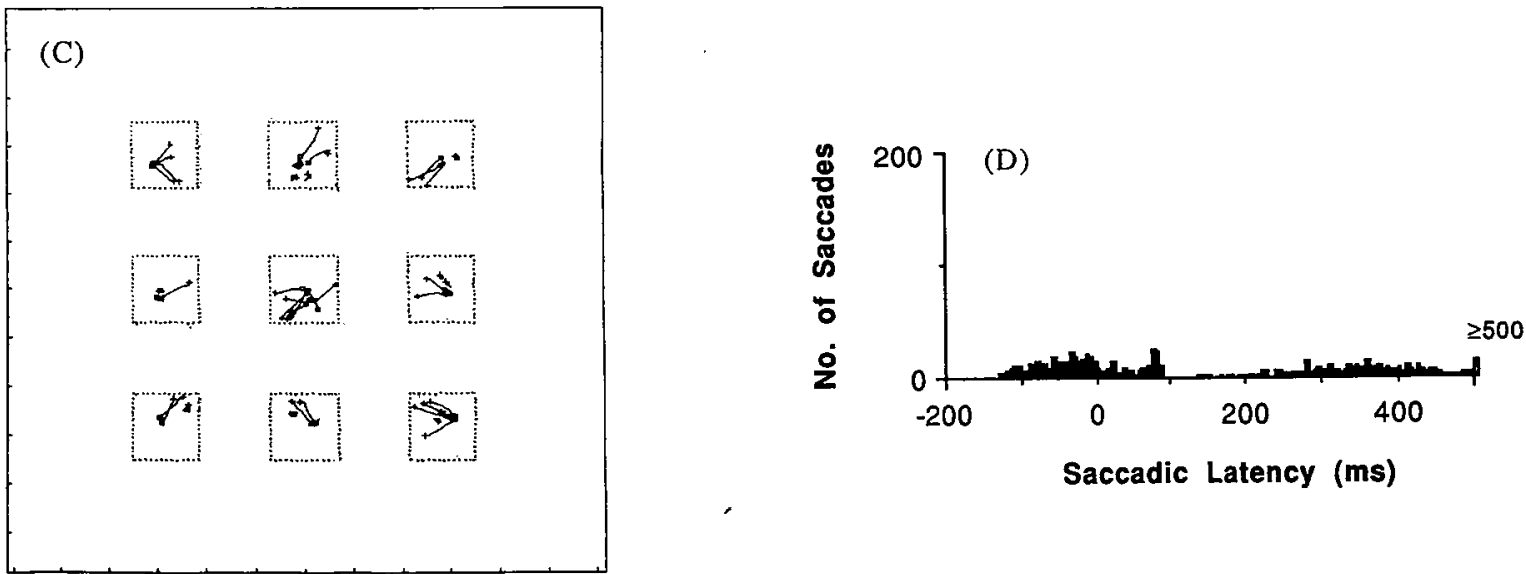

FIGURE 6. Trajectories and latencies of wrong saccades. (A) During the Visual Scan task, these movements were nearly all scanning saccades. (B) The latency histogram of these saccades shows that scanning stopped around 60 msec following target onset, as was seen in Fig. 4. Most of the saccades that resumed about $150 \mathrm{msec}$ later terminated off the visible display [one such saccade is present in the upper left quadrant of (A)]. (C) In the Steady Gaze task, wrong saccades were mostly small movements that corrected for inaccurate initial fixation. (D) Latency distribution of wrong saccades in the Steady Gaze task. Saccade traces in (A) and (C) are of monkey "D", all $150 \mathrm{msec}$ target duration trials. Histograms in (B) and (D) pool together data from both monkeys, all target onset lags, and all target durations. 
Visual scan

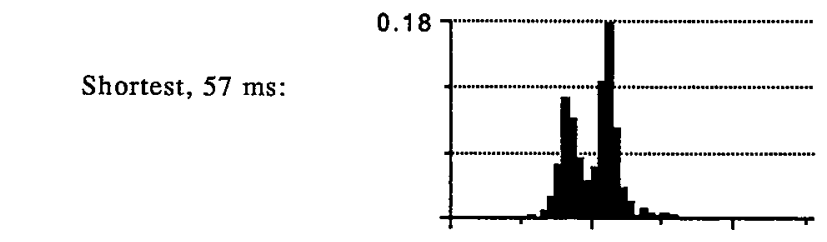

Steady gaze

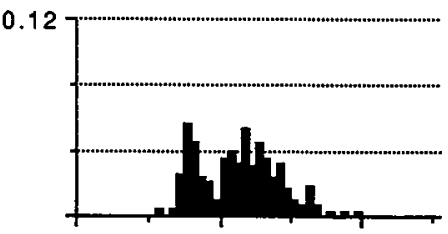

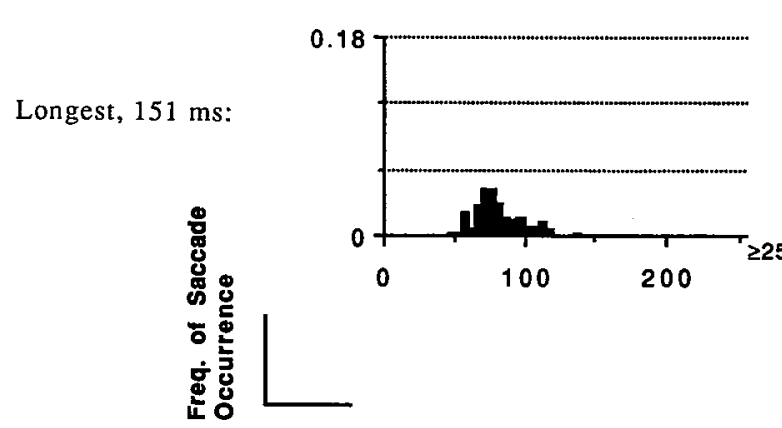

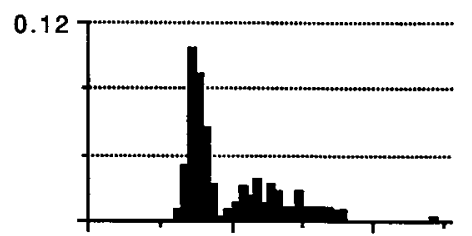

Saccadic Latency (ms)

FIGURE 7. Variation in latency distributions of correct saccades with target onset lag (monkey " $D$ ", all target durations pooled). Left column, Visual Scan task; right column, Steady Gaze task. Top row, shortest target onset lag (57 msec); bottom row, longest target onset lag $(151 \mathrm{msec})$. In either task, longer target onset lags favor express saccade occurrence. Ordinate of histograms is in frequency of occurrence, the number of saccades divided by the number of target presentations (300-600 target presentations per histogram).

\section{DISCUSSION}

The four main findings of this study will be discussed first, with emphasis placed on the Visual Scan task results. Drawing on these results and other recent findings, a physiological basis for express saccades will then be proposed.

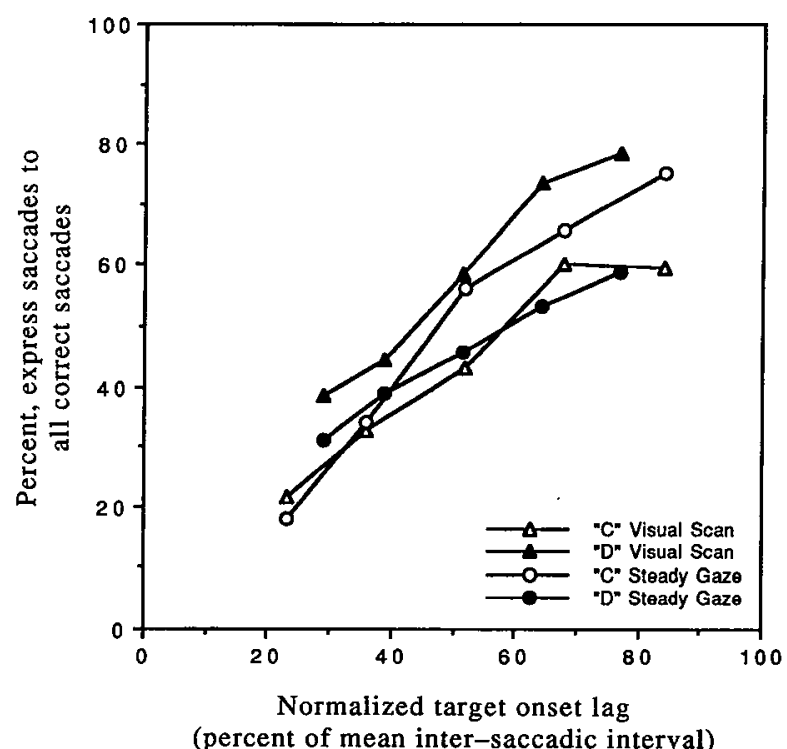

FIGURE 8. Target onset lag effect for both monkeys in the Visual Scan and Steady Gaze tasks. The percent of correct saccades that have express latency always rises with increasing target onset lag. This study's convention for calculating percentages is that latencies of $<95 \mathrm{msec}$ are considered express, and latencies of $\geqslant 95 \mathrm{msec}$ are regular. On abscissa, target onset lags are normalized as a percent of each monkey's mean inter-saccadic interval.

\section{Proximity transition with latency}

During a narrow transition region, the distance between saccadic endpoints and the suddenly appearing target decreased $75 \%$ in the Visual Scan task, from an average of about $8 \mathrm{deg}$ proximity to the target to $<2 \mathrm{deg}$ proximity. Notably, the transition zone of the present study was only about $20 \mathrm{msec}$ wide even though the target could randomly appear at any of five times following the start of fixation, over a total span of more than $90 \mathrm{msec}$. This strongly suggests that the proximity transition was synchronized to the onset of the suddenly appearing target, and not to any other event, such as start of fixation.

It was also quite interesting that the proximity transition from 50 to $70 \mathrm{msec}$ latency was gradual in the Visual Scan task. Saccades with latencies that were within the proximity transition zone tended to exhibit spatial averaging. That is, these transition zone saccades fell closer to the suddenly appearing target than the earlier, purely scanning, saccades, but they were not as accurate as the later express and regular mode saccades (Fig. 4). Hence, averaging saccades are not only made to flashed targets separated in time (Becker \& Jürgens, 1979) and to flashed targets presented simultaneously but separated in space (Findlay, 1982), but they can also be made toward a target flashed in a field that includes other, continually present, visual stimuli.

Therefore, this result demonstrates how the monkey saccadic eye movement system reacts to the sudden appearance of a behaviorally significant visual stimulus during a saccadic scan of visual structure. Scanning saccades are halted shortly after the onset of the flashed stimulus. During a $20 \mathrm{msec}$ transition period, saccades land in averaged positions between the structural stimuli 
Visual scan

Shortest, $33 \mathrm{~ms}$ :

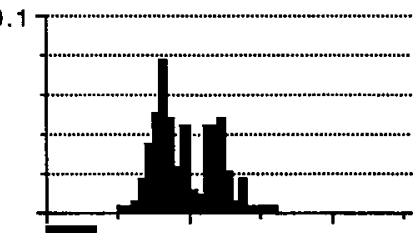

Steady gaze

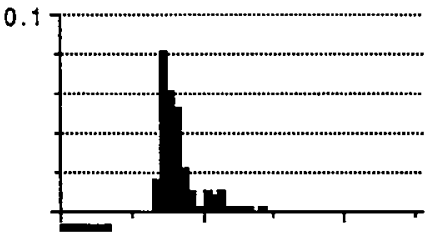

Longest, $150 \mathrm{~ms}$ :

0.1
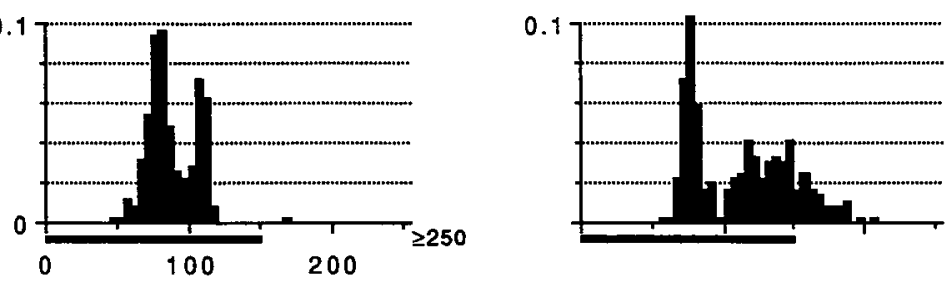
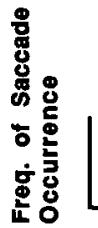

\section{Saccadic Latency (ms)}

FIGURE 9. Variation in latency distributions of correct saccades with target duration (monkey "D", all target onset lags pooled). Top row, shortest target duration $(33 \mathrm{msec})$; bottom row, longest target duration $(150 \mathrm{msec})$. Longer target durations increase regular mode occurrence in the Steady Gaze task (right), without affecting the express mode. Target duration had little effect on Visual Scan task distributions (left) for this monkey. Target duration is represented by a bar below the abscissa of each histogram.

and the suddenly appearing target. Saccades that appear after this transition land at the suddenly appearing target location with high accuracy. Since the cessation of scanning occurs at around $60 \mathrm{msec}$ latency, the latency at which express saccades appear in the monkey, this effect might be termed "express" scanning suppression.
Visual scan

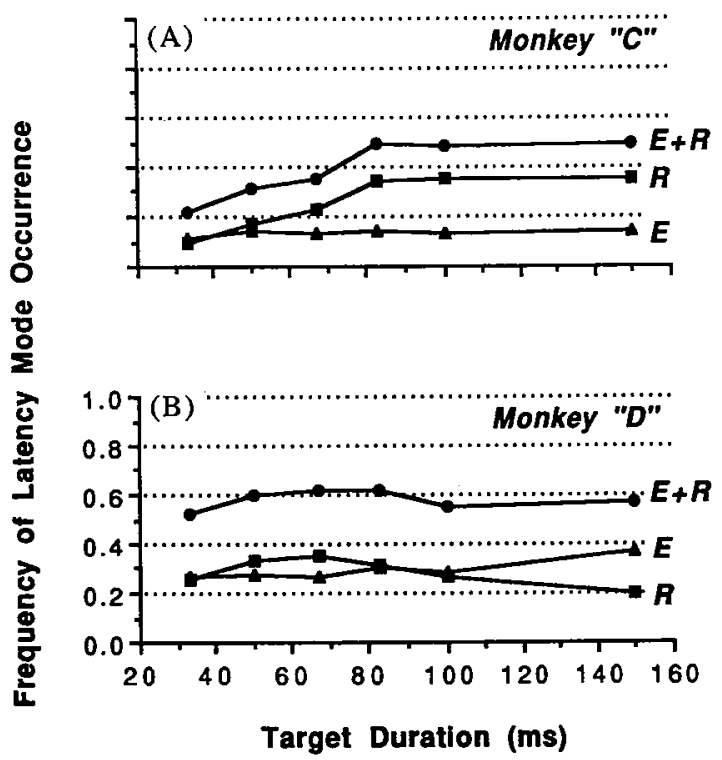

Steady gaze
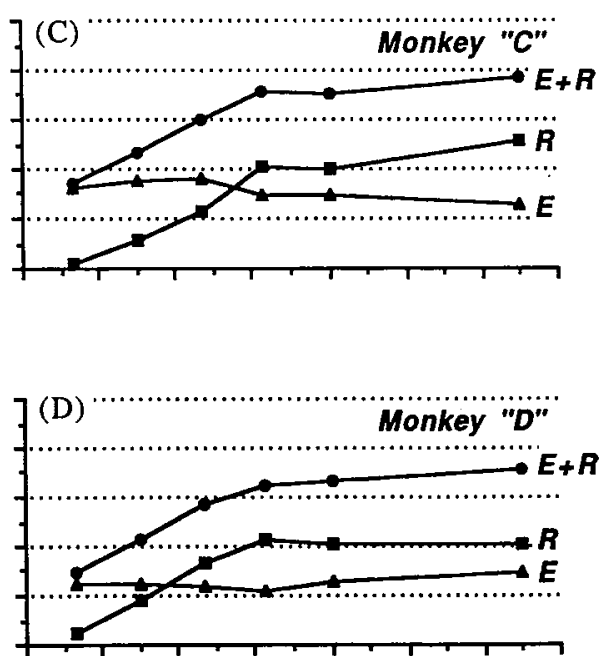

FIGURE 10. Quantitative summary of the target duration éffect. Each datum is the frequency. of occurrence of an express (triangles) or regular (squares) saccade, obtained by integrating under the express mode (by convention, from 0 to $95 \mathrm{msec}$ ) and regular mode $(\geqslant 95 \mathrm{msec})$ of histograms such as those in Fig. 9. The data are connected to form $\mathrm{E}$ and $\mathrm{R}$ curves vs target duration. $\mathrm{E}+\mathrm{R}$ curve (circles) is the sum of the express and regular mode curves, which gives the overall frequency of correct saccades. In the Steady Gaze task (right), saccades of the regular mode ( $\mathrm{R}$ curve) become increasingly frequent as target duration is increased, for both monkeys (C, D); this causes a doubling in the probability of evoking correct saccades in general ( $\mathrm{E}+\mathrm{R}$ curve), from a frequency between 0.3 and 0.4 at $33 \mathrm{msec}$ target duration to a frequency between 0.7 and 0.8 at $150 \mathrm{msec}$ target duration. The frequency of express saccades always remains relatively unchanged with target duration. During the Visual Scan task (left), monkey "C" (A) shows an increase in regular saccades from 33 to $83 \mathrm{msec}$, but this is not seen with monkey "D" (B). 

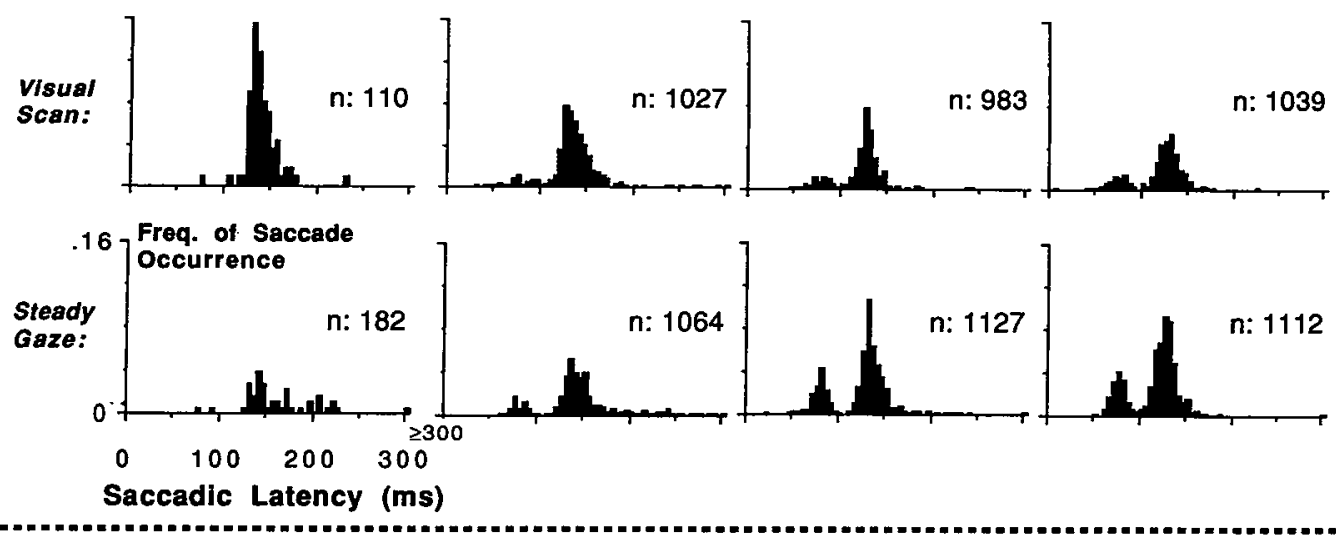

(B)

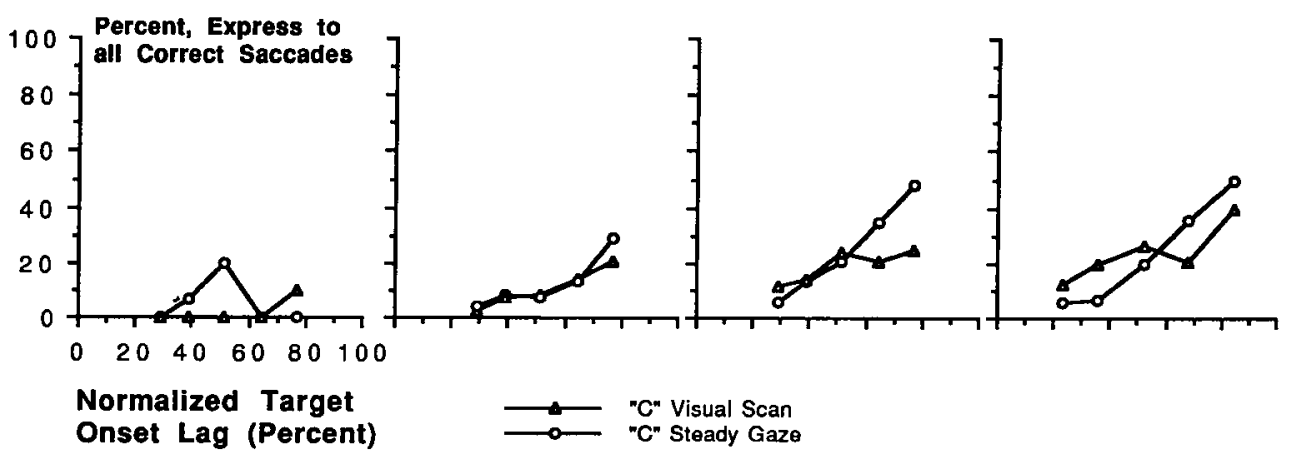

(C)

Visual

Scan:

Gaze:
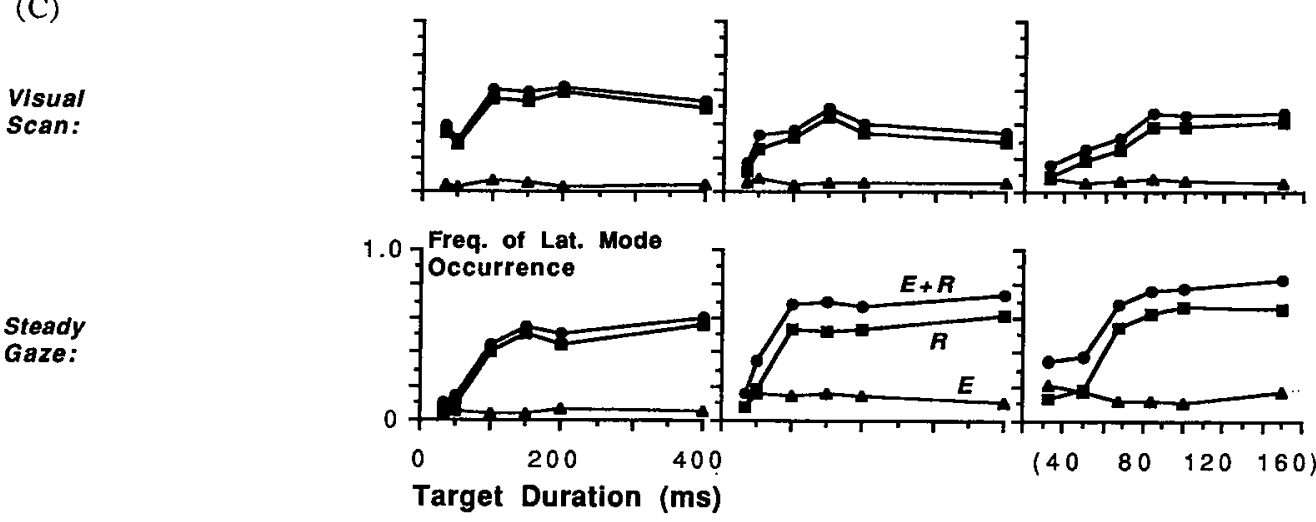

FIGURE 11. Evolution of saccadic latency bimodality, the target onset lag effect, and the target duration effect with short-term training (monkey "C"). The first three days of this animal's training are presented, and then day 7 is shown, in separate columns. (A) An express mode is seen on day 2 for both tasks, and it becomes more prominent with further training. $n$, number of trials in which the target was presented (non-aborted trials) for each task per day. (B) The target onset lag effect (see Fig. 8) is present on day 2 for both tasks. (B) The target duration effect (see Fig. 10) also was strong from day 2 onward. Variation in target duration was not tested on day 1 . Target onset lags were shortened slightly from day 3 to day 7 to account for shortened mean of the inter-saccadic interval distribution that occurred during this time (see Fig. 12). A change in target durations that occurred on day 6 is indicated parenthetically on day 7 .

\section{Bimodal latency distributions during the Visual Scan task}

The second main finding of this paper is that the latency distribution of correct, target-directed saccades was bimodal in the Visual Scan task, forming separate express and regular modes. This was surprising, considering that two conditions inherent in visual scan should supposedly be disadvantageous for eliciting express saccades. First, visual scan conditions are "overlap", since the spot that is foveated does not disappear before target onset. Only two other studies have reported success at eliciting express saccades during overlap in the monkey (Rogal, Reible \& Fischer, 1985; Boch \& Fischer, 1986). This confirms those studies and also shows that express saccades can occur in the overlap condition even during extensive randomization and after very little training. Second, at the moment of target presentation there were a total of 10 stimuli present on the display ( 1 target +9 
fixation stimuli). It has been shown that high-contrast distractors inhibit express saccades if their onset is simultaneous with the target's onset (Schiller et al., 1987; McPeek, Sommer \& Schiller, 1991). The present report extends these findings to show that there are conditions in which non-target visual stimuli do not inhibit express saccades: if they are much different in appearance from the target and are present continuously, non-target stimuli have little, if any, inhibitive effect.

\section{Target onset lag effect}

The present study demonstrates that express saccades are inhibited at short target onset lags, during both Visual Scan and the more traditional Steady Gaze task, and that this effect is present as soon as express saccades appear during training. There are at least three possible explanations for this effect. First, it can be presumed that a scanning saccade is being prepared during each fixation in the Visual Scan task. If computation of its endpoint metrics is altered by the suddenly appearing target, the resulting saccade might be mistaken for an express saccade that had been prepared exclusively in response to the target. Since the likelihood of scanning saccade initiation increases as the fixation progresses, the likelihood of an express saccade, supposedly derived from the partially prepared scanning saccade signal, should also increase as fixation progresses. This explanation fails, however, because the target onset lag effect is as strong in the Steady Gaze task as in the Visual Scan task (Fig. 8). In the Steady Gaze task, the monkey does not produce, and thus presumably does not prepare, scanning saccades; rather, the monkey steadily fixates a single point. Yet express saccades occur in abundance, with the probability of their occurrence increasing with target onset lag as steeply as in the Visual Scan task.

A second possible explanation for the target onset lag effect focuses on the temporal intervals that were chosen for the five target onset lags. In the present study, five lags were chosen, spaced by $20-25 \mathrm{msec}$. The conditional probability of target appearance rises if fixation progresses and the target has not yet appeared, from 0.20 at the start of fixation to 1.0 after the fourth target onset lag has elapsed. It is possible that a subject takes advantage of this change in conditional probability, decreasing its saccadic latency through an overall increase in arousal due to expectation that a target is more likely to appear as fixation progresses. More research is
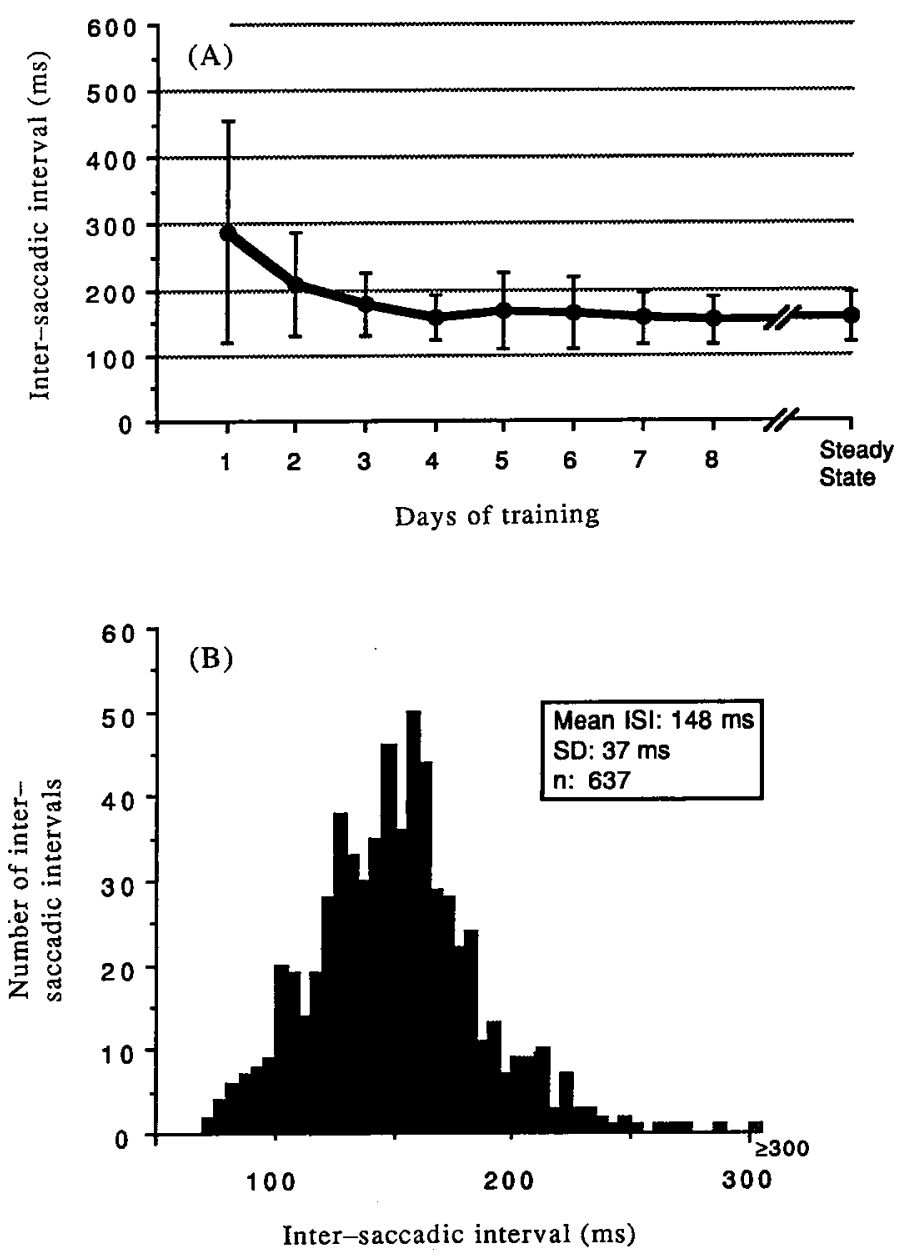

FIGURE 12. (A) Inter-saccadic intervals became shorter and less variant as training progressed (monkey "C"). (B) Typical inter-saccadic interval distribution for one session of five blocks of testing in the Visual Scan task at steady state (monkey " $\mathrm{C}$ ", target duration $67 \mathrm{msec}$ ). Inter-saccadic interval (ISI) histograms were always unimodal for both monkeys, even though they often encroached into the "express" range of latencies (below $95 \mathrm{msec}$ or so). SD, standard deviation; $n$, total number in histogram. 
needed to directly test whether the target onset lag effect on express saccades is as strong with schedules of target delays for which conditional probability is constant with time (Hanes, Tu \& Schall, 1992). It does not appear, however, that the monkeys in this study used the rise in conditional probability to their advantage, at least in the Visual Scan task. The scanning strategy that is most strategic, if there is awareness of the increase in conditional probability of target onset with progression of fixation, is to continue foveating each fixation stimulus until the longest target onset lag has elapsed and only then scan to the next fixation stimulus. Terminating the fixation before the longest target onset lag elapses is a disadvantageous strategy that results in a high percentage of wrong saccades and a lower juice reward frequency; yet, this strategy is exactly what is implemented by the monkeys in this study. Since the longest target onset lag for each monkey is set at about $80 \%$ of the animal's mean inter-saccadic interval, on approx. $40 \%$ of trials the animal breaks fixation before the longest target onset lag could elapse. To take advantage of rising conditional probability with time, a monkey would need to increase the mean of its inter-saccadic interval distribution, i.e. scan at a slower rate, after the target onset lag values were set in early training. This was never observed (Fig. 12).

A third explanation, which seems most consistent with the data, is that after start of fixation there is a lessening of inhibition on the neural mechanisms that generate express saccades. The express saccade is not partially prepared beforehand, as is implied by the first explanation, above, but it is fully prepared after the target's abrupt onset; the mechanisms that allow this express saccade preparation simply become more disinhibited as fixation continues. Since a period of fixation is an event that is common to both the Visual Scan and Steady Gaze tasks, the fact that the target onset lag effect is equally strong in both tasks is to be expected. This explanation of the target onset lag effect therefore supports those hypotheses of express saccade production for which disinhibition of saccadic mechanisms with continued fixation is a major component, such as the "disengagement" of attention hypothesis (Fischer \& Breitmeyer, 1987) and the "visuomotor" physiological idea (Rohrer \& Sparks, 1986) that is revived below. This third explanation is less compatible with other hypotheses, such as those which propose that express saccades are generated by triggering a buffered "motor program" (reviewed by Kowler, 1990); such hypotheses rely on the presence of partial saccade preparation before target onset and an anticipation that the target is about to appear, i.e. they depend on the validity of the first two explanations, above.

Finally, it is remarkable that the rise in percent of express saccades with target onset lag is so steep (Fig. 8). This increase parallels the fast rise in percent of express saccades elicited when varying the gap duration from 0 to $200 \mathrm{msec}$ in the gap paradigm (Mayfrank et al., 1986; Schiller et al., 1987). Further work is needed to deter- mine if this is circumstantial or reflects a true relation between the effects.

\section{Target duration effect}

The target duration effect in the Steady Gaze task is similar to results of step-return paradigms, in which a fixated target is briefly stepped to a new location, but then re-appears at the original position (Westheimer, 1954; Komoda, Festinger, Phillips, Duckman \& Young, 1973; Becker \& Jürgens, 1979). In the Steady Gaze task, the "stepped" target is physically different from the fixation stimulus, and the fixation stimulus does not momentarily disappear, but the results with respect to "step" duration are similar. Komoda et al. (1973) found that the probability that a human subject would make a saccade in response to the step increased as a function of the step duration; the present study found that the probability that a monkey would make a saccade to a target flash increased as a function of the target duration. The curves of Fig. 10, Steady Gaze task, are quite similar to those of Komoda et al. (1973, their Fig. 1). These results therefore seem to extend their finding to show that the regular mode of a bimodal distribution is affected by the "step" duration, but not the express mode. Why the effect is so prominent in the Steady Gaze task but not in the Visual Scan task may be related to the presence of visual structure in the latter task.

In this report it was also found that the target duration effect diminished with extensive training. It is possible that the animals used by Boch et al. (1984) were too highly trained to allow them to find an effect of target duration. Also, these authors emphasized how target parameters affected express saccades, but it is the regular mode that is strongly dependent on target duration.

Why consider a possible physiological basis for express saccades?

This report has demonstrated that express saccades can be elicited to a suddenly appearing target presented during visual scan, with very little training, indicating that a bimodal distribution of saccadic latencies may be a natural response to suddenly appearing visual stimuli. To progress in an understanding of this phenomenon, it is necessary to present detailed, testable hypotheses that are compatible both with the present and prior psychophysics results and with established physiological findings. The remainder of this discussion will focus on one such hypothesis.

\section{Visuomotor hypothesis of express saccades}

Recent lesion work (Schiller et al., 1987; Schiller \& Lee, 1994; reviewed by Sommer, Schiller \& McPeek, 1994) indicates that an intact superior colliculus (SC) is necessary for the generation of both the express and regular latency modes. It is known that many neurons in the intermediate and deeper layers of the SC respond to visual stimuli with a dual, temporally separate discharge (Schiller \& Koerner, 1971; Wurtz \& Goldberg, 1972; Schiller \& Stryker, 1972). The first, phasic, burst occurs 
$40-50 \mathrm{msec}$ after a visual stimulus appears in a cell's receptive field (Wurtz \& Goldberg, 1972); the later, more sustained discharge, has been correlated with saccade onset. Regardless of the "message" supposedly carried by these two discharges, they are both just trains of action potentials that share a common neural conduit, the visuomotor cell. Either source of discharge could theoretically cause a saccade command that could be used downstream to initiate saccadic behavior (noted by Sparks \& Hartwich-Young, 1989). This leads to two proposals:

(1) the "visual" response of the superior colliculus visuomotor cells is necessary for express mode saccades;

(2) the "motor" response of these cells is necessary for regular mode saccades.

Another property of the SC is that it is subject to fixation-dependent inhibition. For example, the amount of electrical stimulation needed to evoke a saccade in the $\mathrm{SC}$ increases if a subject's fixation is required to be more intent (Schiller \& Sandell, 1983; Sparks \& Mays, 1983). This inhibition seems to have at least two sources: the substantia nigra pars reticulata (SNr) (Hikosaka \& Wurtz, 1983d) and the rostral region of the superior colliculus (Munoz \& Wurtz, 1992). Cells of the $\mathrm{SNr}$ release their tonic inhibition of the $\mathrm{SC}$ in response to extra-foveal stimuli (Hikosaka \& Wurtz, 1983a), states of fixation (Hikosaka \& Wurtz, 1983b), and higher level mechanisms such as memory (Hikosaka \& Wurtz, 1983c) and preparation for movement (Schultz, 1986). The rostral SC, serving foveal visual space, appears to exert an inhibition onto the extrafoveal SC that, for many of its cells, is elevated at the start of a fixation period and then steadily declines (Munoz \& Wurtz, 1992). These findings lead to a third proposal:

(3) the selection of which discharge of the visuomotor cells will cause a saccade command is mediated by inhibition of the superior colliculus; this inhibition is elevated at the start of a fixation and decreases thereafter.

These three proposals form a "visuomotor" hypothesis of express saccades. They relate to discharges at the level of the deeper superior colliculi; issues such as the cortical sources of the collicular "visual" and "motor" discharges are beyond the scope of this hypothesis.

\section{Implications of the visuomotor hypothesis}

There are several psychophysical and physiological results that are expected if the proposals of the visuomotor hypothesis are valid; some were found to be true in the present study, others have already been reported, while others serve as predictions. Verification or contradiction of these expected results can serve to test the hypothesis.

(a) Express saccades only to suddenly appearing targets. The visual discharge of a SC visuomotor neuron is phasic, and the receptive field of such a cell overlaps with it movement field (Wurtz \& Goldberg, 1972). Thus, a prediction based on proposal (1) of the visuomotor hypothesis is that express saccades should only be possible if a suddenly appearing stimulus serves both as the cue when to move and where to move. Some support for this has already been reported: express saccades do not appear if the cue when to move is an event other than the target's onset (Boch \& Fischer, 1986); neither do they appear if a visual stimulus cues when to move, but the saccade is required to go away from it (Fischer \& Weber, 1992).

A related consequence of proposal (1) is that an express mode should not be present in the latency distribution of saccades made to continually present targets, e.g. scanning saccades. Although the appearance of a continually present stimulus in a visuomotor cell's receptive field at the start of a fixation would cause a visual discharge, proposal (3) of the visuomotor hypothesis predicts that elevated inhibition at that moment would keep the discharge from initiating an express mode saccade. Thus, scanning saccades should have unimodal latencies, as was found in the present study (Fig. 12), since they can be generated only by the later, motor discharge.

(b) Target onset lag effect. Proposals (1) and (3) of the visuomotor hypothesis imply that as targets are presented later in the fixation period, the probability of evoking express mode saccades should increase. This agrees with the findings of a target onset lag effect in this report.

(c) Target duration effect. Proposal (2) of the visuomotor hypothesis states that the "motor" discharge of the SC visuomotor cells is necessary for regular mode saccades. Because the appearance of regular mode saccades is dependent on the duration of the suddenly appearing target, as found in the present study, it follows that such target duration shortening should affect the "motor" discharge of SC visuomotor cells, leaving the "visual" discharge unaffected. Prior work in the monkey suggests that this is the case (Sparks, 1978), but more detailed study of the physiological basis of the target duration effect is still needed.

(d) Superior colliculus inhibition. If one records from fixation-dependent $\mathrm{SNr}$ or rostral $\mathrm{SC}$ cells during conditions used in this report, one would expect to see a decline in activity synchronized to the start of fixation (as reported by Munoz \& Wurtz, 1992). The visuomotor hypothesis further predicts that, during fixation periods, lower initial discharges and faster rates of decline in fixation-dependent rostral $\mathrm{SC}$ or $\mathrm{SNr}$ units should be correlated with increased probability of observing an express saccade. Another testable prediction is that all experimental conditions that are conducive to producing express saccades should also lower the current thresholds for evoking saccades with electrical stimulation in the $\mathrm{SC}$.

(e) Superior colliculus visuomotor cells. Electrophysiological recording of SC visuomotor cells should find activity related to appearance of express saccades. Rohrer and Sparks (1986) concluded that the deeper layer SC visual discharge does not simultaneously serve, 
unmodified, as the express saccade command. A more recent abstract (Edelman \& Keller, 1993) seems to agree that the deeper layer $\mathrm{SC}$ visual response is not the express saccade command, per se, but it suggests that the visual response does contribute in the formation of a saccadic command. This latter report seems to support the visuomotor hypothesis presented here.

\section{CONCLUSION}

This report presents the first quantified evidence that express and regular saccadic latency modes can be generated to targets that are suddenly presented during visual scan. The sudden appearance of a visual target not only elicits an express or regular mode saccade toward its location, but also suppresses scanning saccades at express latency. That the present findings are reported for the monkey should encourage further physiological study of express saccades in this primate. The present findings and recent physiological results together support a visuomotor hypothesis of express saccades. Several testable predictions can be derived from the hypothesis. If the visuomotor hypothesis is found to be valid, further, investigation of the rapid generation of saccade commands from the visual responses of SC visuomotor cells will provide a unique opportunity for isolating and understanding a sensorimotor transformation of the primate saccadic eye movement system.

\section{RAFERENCES}

Bahill, A. T., Adler, D. \& Stark, L. (1975). Most naturally occurring saccades have amplitudes of 15 degrees or less. Investigative Ophthalmology and Visual Science, 14, 468-469.

Becker, W. \& Jürgens, R. (1979). An analysis of the saccadic system by means of double step stimuli. Vision Research, 19, 967-983.

Boch, R. \& Fischer, B. (1986). Further observations on the occurrence of express-saccade in the monkey. Experimental Brain Research, 63, 487-494.

Boch, R., Fischer, B. \& Ramsperger, E. (1984). Express-saccades of the monkey: Reaction times versus intensity, size, duration, and eccentricity of their targets. Experimental Brain Research, 55, 223-231.

Carpenter, R. H. S. (1988). Saccades. In Movements of the eyes (p. 83). London: Pion Press.

Edelman, J. A. \& Keller, E. L. (1993). Visual activity in the deeper superior colliculus contributes to the initiation of express saccades. Investigative Ophthalmology and Visual Science (Suppl.), 34, 1137.

Findlay, J. M. (1982). Global visual processing for saccadic eye movements. Vision Research, 22, 1033-1045.

Fischer, B. \& Boch, R. (1983). Saccadic eye movements after extremely short reaction times in the monkey. Brain Research, 260, 21-26.

Fischer, B. \& Breitmeyer, B. (1987). Mechanisms of visual attention revealed by saccadic eye movements. Neuropsychologia, 25, 73-83.

Fischer, B. \& Ramsperger, E. (1984). Human express saccades: Extremely short reaction times of goal directed eye movements. Experimental Brain Research, 57, 191-195.

Fischer, B. \& Ramsperger, E. (1986). Human express saccades: Effects of randomization and daily practice. Experimental Brain Research, 64, 569-578.

Fischer, B. \& Weber, H. (1992). Characteristics of "anti" saccades in man. Experimental Brain Research, 89, 415-424.

Fischer, B., Weber, H. \& Biscaldi, M. (1993). The role of prediction in the generation of express saccades. Investigative Ophthalmology and Visual Science (Suppl.), 34, I137.
Fischer, B., Boch, R. \& Ramsperger, E. (1984). Express-saccades of the monkey: Effect of daily training on probability of occurrence and reaction time. Experimental Brain Research, 55, 232-242.

Fuchs, A. F. (1967). Saccadic and smooth pursuit eye movements in the monkey. Journal of Physiology, 191, 609-631.

Fuchs, A. F. \& Robinson, D. A. (1966). A method for measuring horizontal and vertical eye movement chronically in the monkey. Journal of Applied Physiology, 21, 1068-1070.

Hanes, D., Tu, F. \& Schall, J. (1992). Effects of temporal context (conditional probability) on saccade latency in the macaque. Society for Neuroscience Abstracts, 18, 698.

Hikosaka, O. \& Wurtz, R. H. (1983a). Visual and oculomotor functions of monkey substantia nigra pars reticulata-I. Relation of visual and auditory responses to saccades. Journal of Neurophysiology, 49, 1230-1253.

Hikosaka, O. \& Wurtz, R. H. (1983b). Visual and oculomotor functions of monkey substantia nigra pars reticulata-II. Visual responses related to fixation of gaze. Journal of Neurophysiology, 49, 1254-1267.

Hikosaka, O. \& Wurtz, R. H. (1983c). Visual and oculomotor functions of monkey substantia nigra pars reticulata-III. Memorycontingent visual and saccade responses. Journal of Neurophysiology, 49, 1268-1284.

Hikosaka, O. \& Wurtz, R. H. (1983d). Visual and oculomotor functions of monkey substantia nigra pars reticulata-IV. Relation of substantia nigra to superior colliculus. Journal of Neurophysiology, 49, 1285-1301.

Jüttner, M. \& Wolf, W. (1992). Occurrence of human express saccades depends on stimulus uncertainty and stimulus sequence. Experimental Brain Research, 89, 678-681.

Kalesnykas, R. P. \& Hallett, P. E. (1987). The differentiation of visually guided and anticipatory saccades in gap and overlap paradigms. Experimental Brain Research, 68, 115-121.

Komoda, M. K., Festinger, L., Phillips, L. J., Duckman, R. H. \& Young, R. A. (1973). Some observations concerning saccadic eye movements. Vision Research, 13, 1009-1020.

Kowler, E. (1990). The role of visual and cognitive processes in the control of eye movement. In Kowier, E. (Ed.), Reviews of oculomotor research, Vol. 4: Eye movements and their role in visual and cognitive processes (pp. 52-54). Amsterdam: Elsevier.

Mayfrank, L., Mobashery, M., Kimmig, H. \& Fischer, B. (1986). The role of fixation and visual attention in the occurrence of express saccades in man. European Archives in Psychiatry and Neurological Sciences, 235, 269-275.

McPeek, R. M., Sommer, M. A. \& Schiller, P. H. (1991). Saccadic latencies to stimuli defined only by luminance, chrominance, motion, and depth. Society for Neuroscience Abstracts, 17, 847.

Munoz, D. P. \& Wurtz, R. H. (1992). Role of the rostral superior colliculus in active visual fixation and execution of express saccades. Journal of Neurophysiology, 67, 1000-1002.

Nothdurft, H. C. \& Parlitz, D. (1993). Absence of express saccades to texture or motion defined targets. Vision Research, 33, 1367-1383.

Robinson, D. A. (1963). A method of measuring eye movement using a scleral search coil in a magnetic field. IEEE Transmissions on Biomedical Engineering, 10, 137-145.

Rogal, L., Reible, G. \& Fischer, B. (1985). Reaction times of the eye and the hand of the monkey in a visual reach task. Neuroscience Letters, 58, 127-132.

Rohrer, W. H. \& Sparks, D. L. (1986). Role of superior colliculus in the initiation of express saccades. Investigative Ophthalmology and Visual Science, 27, 156.

Schiller, P. H. \& Koerner, F. (1971). Discharge characteristics of single units in superior colliculus of the alert rhesus monkey. Journal of Neurophysiology, 34, 920-936.

Schiller, P. H. \& Lee, K.-M. (1994). The effects of lateral geniculate nucleus, area V4, and middle temporal (MT) lesions on visually guided eye movements. Visual Neuroscience, 11, 229-241.

Schiller, P. H. \& Sandell, J. H. (1983). Interactions between visually and electrically elicited saccades before and after superior colliculus and frontal eye field ablations in the rhesus monkey. Experimental Brain Research, 49, 381-392. 
Schiller, P. H. \& Stryker, M. (1972). Single-unit recording and stimulation in superior colliculus of the alert rhesus monkey. Journal of Neurophysiology, 35, 915-924.

Schiller, P. H., Sandell, J. H. \& Maunsell, J. H. R. (1987). The effect of frontal eye field and superior colliculus lesions on saccadic latencies in the rhesus monkey. Journal of Neurophysiology, 57, 1033-1049.

Schiller, P. H., True, S. D. \& Conway, J. L. (1980). Deficits in eye movements following frontal eye-field and superior colliculus ablations. Journal of Neurophysiology, 44, 1175-1189.

Schultz, W. (1986). Activity of pars reticulata neurons of monkey substantia nigra in relation to motor, sensory, and complex events. Journal of Neurophysiology, 55, 660-677.

Smit, A. C. \& Van Gisbergen, J. A. M. (1989). A short-latency transition in saccade dynamics during square-wave tracking and its significance for the differentiation of visually-guided and predictive saccades. Experimental Brain Research, 76, 64-74.

Sommer, M. A. \& Schiller, P. H. (1992). Express saccades elicited during natural fixations of visual search. Society for Neuroscience Abstracts, 18, 1398.

Sommer, M. A., Schiller, P. H. \& McPeek, R. M. (1994). What neural pathways mediate express saccades? Behavioral and Brain Sciences, $16,589-590$.

Sparks, D. L. (1978). Functional properties of neurons in the monkey superior colliculus: Coupling of neuronal activity and saccade onset. Brain Research, 156, 1-16.

Sparks, D. L. \& Hartwich-Young, R. (1989). The deep layers of the superior colliculus. In Wurtz, R. H. \& Goldberg, M. E. (Eds),
Reviews of oculomotor research Vol. 4: The neurobiology of saccadic eye movements (p. 230). Amsterdam: Elsevier.

Sparks, D. L. \& Mays, L. E. (1983). Spatial localization of saccade targets. I. Compensation for stimulation-induced perturbations in eye position. Journal of Neurophysiology, 49, 45-63.

Weber, H. \& Fischer, B. (1990). Effect of a local ibotenic acid lesion in the visual association area on the prelunate gyrus (area V4) on saccadic reaction, times in trained rhesus monkeys. Experimental Brain Research, 81, 134-139.

Weber, H., Latanov, A. \& Fischer, B. (1993). Context dependent amplitude modulations of express and regular saccades in man and monkey. Experimental Brain Research, 93, 335-344.

Westheimer, G. (1954). Eye movement responses to a horizontally moving visual stimulus. Archives of Ophthalmology, 52, 932-941.

Wurtz, R. H. \& Goldberg, M. E. (1972). Activity of superior colliculus in behaving monkey-III. Cells discharging before eye movements. Journal of Neurophysiology, 35, 575-586.

Acknowledgements - I am grateful to P. H. Schiller for advice and careful critique of this paper, to E. J. Tehovnik, J. A. Edelman, and E. L. Cameron for helpful discussion, and to R. P. Dolan for programming assistance. This work was supported by NIH EY-00676, NIH EY-08502, and a National Science Foundation Graduate Research Fellowship. Preliminary findings have been reported elsewhere (Sommer \& Schiller, 1992). 